\title{
Defensoria Pública e legitimação social do direito: UMA ANÁLISE SOBRE RECONHECIMENTO E PARIDADE PARTICIPATIVA NA TUTELA DE GRUPOS VULNERÁVEIS
}

\author{
Public Defender 's office and SOCial legitimation: \\ AN ANALYSIS ON RECOGNITION AND PARTICIPATIVE PARITY \\ IN THE PROTECTION OF VULNERABLE GROUPS
}

Cristian Patric de Sousa Santos

Mestre em Direito Público pela Universidade Federal da Bahia. Servidor

Público Federal do Tribunal Regional Eleitoral da Bahia. ORCID: [https://orcid.org/0000-0003-0805-0849]. cristianpatricsousa@hotmail.com

\section{Ricardo Maurício Freire Soares}

Doutor em Direito pela Università del Salento. Doutor em Direito Público com Pós-Doutorado em Direito pela Università degli Studi di Roma La Sapienza, pela Università degli Studi di Roma TorVergata e pela Università del Salento. Mestre em Direito Privado pela Universidade Federal da Bahia. Professor

da Faculdade de Direito da Universidade Federal da Bahia, Faculdade Baiana de Direito e Uniruy. ORCID: [https://orcid.org/0000-0002-0806-8603]. ric.mauricio@ig.com.br

\author{
Recebido em: 21.05.2020 \\ Aprovado em: 21.09 .2020 \\ DOI: [https://doi.org/10.48143/rdai/17.cpss]
}

ÁrEA DO DIREITO: Constitucional

Resumo: 0 presente artigo pretende avaliar o papel das Defensorias Públicas na tutela de grupos vulneráveis como contributo para a legitimação social do Direito. A partir da análise da luta pelo reconhecimento proposta por Axel Honneth e a releitura proposta por Nancy Fraser sobre reconhecimento e redistribuição, analisa-se a Defensoria Pública como órgão que representa paridade participativa na construção de narrativas emancipatórias de grupos estigmatizados, alijados de processos de participação política.

\begin{abstract}
This paper aims to evaluate the role of Public Defenders in the protection of vulnerable groups as a contribution to the social legitimation of law. From the analysis of the struggle for recognition proposed by Axel Honneth and the reinterpretation proposed by Nancy Fraser about recognition and redistribution, the Public Defender's Office is analyzed as an organ that represents participatory parity in the construction of emancipatory narratives of stigmatized groups, alienated from participation processes politics.
\end{abstract}


Palavras-Chave: Grupos Vulneráveis - Reconhecimento - Redistribuição - Paridade Participativa - Defensoria Pública.
Kerwords: Vulnerable Groups - Recognition Redistribution - Participatory Parity - Publica Defender.

SUMÁRI0: 1. Introdução. 2. Direito, luta pelo reconhecimento e paridade participativa: uma breve análise em Axel Honneth e Nancy Fraser. 3. Construção de narrativas emancipatórias e a legitimação social do direito pela tutela de grupos vulneráveis. 4. A Defensoria Pública e a legitimação social do direito por meio da paridade participativa. 4.1. A Defensoria Pública como via institucional de penetração argumentativa dos grupos vulneráveis aos sistemas judiciais. 5. Considerações finais. 6. Referências bibliográficas.

\section{INTRODUÇÃO}

O presente artigo tem o propósito de avaliar o papel das Defensorias Públicas, instituição de representatividade constitucionalmente prevista para a defesa de pessoas e grupos em situação de vulnerabilidade, na construção da legitimação social do Direito.

O Direito, a princípio, sob a vigência dos discursos da teoria crítica predominante na cultura jurídica brasileira no momento pré-constitucional, era enquadrado como instrumento de dominação, a partir da legitimação de discursos produzidos pelos grupos que acessam, com maior facilidade, os sistemas de justiça.

Com vistas a desconstruir a utilização do Direito como instrumento de dominação enquanto ideologia, o desbloqueio dos canais de acesso à justiça por meio da construção de narrativas emancipatórias engendradas no seio de grupos historicamente estigmatizados será apresentado como fator de legitimação social. A construção de respostas justas às disputas inerentes ao convívio em sociedade depende da influência da argumentação produzida pelos grupos vulneráveis.

Para tanto, o artigo iniciará realizando uma breve revisão bibliográfica, com a análise da luta por reconhecimento de Axel Honneth, cuja tese central afirma que a construção da identidade de um indivíduo ocorre em relação intersubjetiva, por intermédio do mecanismo do reconhecimento, a partir da sua relação com outros membros da sociedade, pelas dimensões do amor, do direito e da solidariedade. A violação de cada uma dessas dimensões básicas da vida humana impulsiona a luta pelo reconhecimento intersubjetivo e social e origina os conflitos sociais. Em seguida, foca-se na releitura proposta por Nancy Fraser, que trata o reconhecimento como questão de justiça, e não como questão de ética, e articula as noções de redistribuição e reconhecimento como complementares.

No tópico seguinte, o artigo analisa a legitimação social do Direito a partir da construção de narrativas emancipatórias engendradas pelos grupos estigmatizados, os quais, por meio da luta pelo reconhecimento e com base na ideia de paridade participativa, tentam acessar os centros decisórios estatais. 
vulnerabilis, ampliam a possibilidade, portanto, de reverberar a argumentação dos grupos vulneráveis nos centros de decisão.

\section{CONSIDERAÇõeS FINAIS}

O presente artigo teve como objetivo, assim, apresentar uma linha argumentativa em defesa das Defensorias Públicas como instrumentos de legitimação social do Direito por intermédio da tutela de grupos vulneráveis. Apresentam-se, de forma sintética, as conclusões finais do trabalho:

1. Os conflitos sociais, na perspectiva de Honneth, são originados na luta pelo reconhecimento intersubjetivo e social, marcados por sentimentos de desrespeito e ofensa ocasionados por violações às dimensões do amor, do reconhecimento jurídico e da solidariedade. A luta por reconhecimento, o impulso para a resistência social e para o conflito, podem ser potencializadas para se elevarem a verdadeiros atos de resistência política, desde que o meio de articulação de um movimento social esteja disponível para tanto, elaborando-se, assim, uma gramática moral dos conflitos sociais.

2. Fraser, em releitura à Honneth, encara as dimensões de redistribuição e reconhecimento como complementares e, a partir da análise de justiça social que exige ambas as dimensões, desenvolve uma tipologia dos movimentos sociais pautados em um modelo bidimensional, situando, de um lado, modos de coletividade associados a modelos redistributivos de justiçae, de outro, modos de coletividade associados a modelos de reconhecimento.

3. Quando permitida a participação de atores sociais na dimensão política da comunidade, verificam-se as condições recíprocas de reconhecimento. Do contrário, quando se estabelece um padrão cultural excludente aos grupos estigmatizados, está-se diante da falta de reconhecimento. Os problemas de reconhecimento, assim, são redirecionados da esfera psicológica para a esfera institucional e a negação do reconhecimento implica violação da justiça. Fraser sustenta, a partir de então, a ideia de participação paritária, ou paridade participativa, para que todos os membros de uma coletividade possam interagir no meio social.

4. A paridade participativa exige a implementação de uma condição objetiva/redistributiva da justiça, mediante a redução das desigualdades sociais e econômicas, e uma condição intersubjetiva/reconhecimento, que exige igual respeito a todos os membros da comunidade e que sejam objeto de estima social.

5. O Direito, ciência social aplicada antes encarada como mero instrumento de legitimação formal do poder, abre-se para a participação de grupos estigmatizados, os quais exercem influência nos centros decisórios estatais a partir da construção de narrativas emancipatórias em prol de grupos vulneráveis. Tal abertura incorpora legitimação social ao Direito, uma vez que teses e/ou antíteses são formadas a partir de/ ou com a utilização dos parâmetros estabelecidos nos argumentos produzidos pelos grupos vulneráveis.

6. As Defensorias Públicas, por meio de instrumentos específicos previstos no Direito brasileiro, garantem acesso institucional dos grupos vulneráveis aos centros decisórios estatais e reverberam, então, os argumentos produzidos por estes grupos nos sistemas de justiça, garantindo-se uma síntese mais justa dos processos dialéticos travados nos sistemas de justiça. 


\section{REFERÊNCIAS BIBLIOGRÁFICAS}

FRASER, Nancy; DAHL, Hanne Marlene; STOLTZ, Pauline; WILLIG, Rasmus. Redistribution and Representation in Capitalist Global Society: An Interview with Nancy Fraser. Acta Sociologica, v. 47, n. 5, 2004.

FRASER, Nancy; HONNETH, Axel. Redistribution or Recognition: a political-philosophical Exchange. Trad. Joel Golb, James Ingram, Christiane Wilke. London, New York: Verso, 2003.

GOMES, Juliana Cesario Alvim. Por um constitucionalismo difuso: cidadãos, movimentos sociais e o significado da Constituição. Salvador: JusPodivm, 2016.

HOBBES, Thomas. Leviatã: ou a matéria, forma e poder de um estado eclesiástico e civil. 3. ed. Trad. Rosina D'Agina. São Paulo: Ícone, 2014.

HONNETH, Axel. Luta por reconhecimento: a gramática moral dos conflitos sociais. Trad. Luiz Repa. São Paulo: Editora 34, 2009.

LASSALE, Ferdinand. O que é uma Constituição? São Paulo: CL Edijur, 2014.

LOCKE, John. Segundo tratado sobre o governo. São Paulo: Martin Claret, 2006.

MARX, Karl. A ideologia alemã. 9. ed. São Paulo: Hucitech, 1993.

RAWLS, John. Umateoria dajustiça. 4. ed. Trad. Álvaro de Vita. São Paulo: Martins Fontes, 2016.

ROUSSEAU, Jean-Jacques. Do Contrato Social. Trad. Pietro Nassetti. São Paulo: Ed. Martin Claret, 2004.

SANTOS, Boaventura de Souza. A crítica da razão indolente: contra o desperdício da experiência. São Paulo: Cortez, 2000.

SCHIER, Paulo Ricardo, Filtragem constitucional: construindo uma nova dogmática jurídica. Porto Alegre: Sergio Antonio Fabris, 1999.

SPINELLI, Leticia Machado. Repensando o reconhecimento: a crítica de Nancy Fraser ao modelo identitário de Axel Honneth. Século XXI, Revista de Ciências Sociais, v. 6, n. 1, p. 204-234, jan.-jun. 2016.

\section{PESQUISAS do EDITORIAL}

\section{Veja também Doutrinas relacionadas ao tema}

- A legitimidade coletiva da defensoria pública para a tutela de segmentos sociais vulneráveis, de Maurilio Casas Maia - RDC 101/351-383 (DTR|2015|16892);

- Defensoria pública e acesso à ordem jurídica justa (K. Watanabe): transversalidade em 6 (seis) ondas renovatórias do acesso à justiça, de Maurilio Casas Maia - RDC 134/427-458 (DTR|2021|6900); e

- 0 percurso histórico da consolidação do direito de acesso igualitário à justiça no Brasil, de Cleber Francisco Alves - RePro 184/329-362 e Doutrinas Essenciais de Direitos Humanos 5/753-784 (DTR|2010|349). 


\section{SUMÁRIO}

LINHA EDITORIAL - EDITORIAL LINE

APRESENTAÇÃO - INTRODUCTION

1. Seção - Direito Administrativo

Section 1 - Administrative LaW

A) Princíplos do Direito Administrativo

A) Principles of Administrative Law

Precedentes administrativos: um caminho para segurança jurídica nas relações entre a Administração Pública e os cidadãos

Administrative precedents: a path to legal security in the relationship between public administration and citizens

Carlos Henrique Benedito Nitão loureiro e Vladimir da Rocha França.....

Ética da discussão e princípios da administração pública: o administrador público mentiroso e a improbidade administrativa Discussion ethics and principles of public administration: the lying public administrator and administrative improbity

Caroline Müller Bitencourt e Janriê Rodrigues Reck.

B) Processo Administrativo

B) Administrative Proceedings

Consensualidade como modo alternativo de exercício da pretensão punitiva estatal no processo administrativo sancionador

Consensuality as an alternative mode for the exercise of state punitive pretension in the administrative sanctional process

Shirlei Silmara de Freitas Mello e Daniela Almeida Campos. 


\section{C) Licitação e Contratos Administrativos \\ C) Bidding and Administrative Contracts}

A consagração popular no procedimento de inexigibilidade para contratação de artistas musicais

The popular consecration in the inexigibility procedure for contracting musical artists

Pedro Henrique Maciel Barros e Marina Zava de Faria

D) Intervenção do Estado na Ordem Social

D) State Intervention in the Social Order

Defensoria Pública e legitimação social do direito: uma análise sobre reconhecimento e paridade participativa na tutela de grupos vulneráveis

Public Defender's office and social legitimation: an analysis on recognition and participative parity in the protection of vulnerable groups

Cristian Patric de Sousa Santos e Ricardo Maurício Freire Soares

\section{SeÇão - Complance}

\section{Section 2 - Compliance}

Compliance e responsabilidade de pessoas jurídicas

Compliance and responsibility of legal entities

Ricardo Marcondes Martins

Compliance ambiental-energético e a ecoeficiência empresarial: cenário na pandemia da Covid-19

Environmental-energy compliance and business eco-efficiency: pandemic scenario of Covid-19

Priscila Elise Alves Vasconcelos, Cleyson de Moraes Mello e Paulo Sérgio

VASCONCELOS

\section{SeÇÃo - Doutrina Estrangeira}

\section{SECTION 3 - FOREIGN DOCTRINE}

Sobre la naturaleza jurídica de los aeropuertos privados internacionales

The legal nature of international private airports

Jaime Rodriguez-Arana Muñoz 
La libre competencia en la compra pública

Free competition in public procurement

Juan David Duque Botero.

El procedimiento sancionadoren la Ley General de Responsabilidades Administrativas

The sanctioning procedure in the General Administrative Responsibilities Act

Manlio Fabio Casarin León.

\section{4a. SeÇão - Pareceres}

\section{Section 4 - Legal Opinions}

Controle do ato discricionário arbitrário na gradação da pena administrativa de multa aplicada pela administração pública federal, atecnia do caput do art. $2^{\circ}$ e inobservância da primariedade do inciso I, da Lei 7.889/1989: desrespeitos aos princípios da motivação, proporcionalidade, razoabilidade e direitos fundamentais da empresa/administrada que pode inviabilizar atividade econômica de empresa de economia familiar protegida pelo artigo 170 da Constituição de 1988

Control of the arbitrary discretionary act in grading the fine administrative penalty applied by the federal public administration, atecnia of the caput of art. 2nd and inobservance of the primariety of item I, of Law 7,889/1989: disrespect to the principles of motivation, proportionality, reasonability and fundamental rights of the company/manager that may invibialize the economic activity of the family economy company protected by article 170 of the 1988 Constitution

Jerson Carneiro Gonçalves Junior

\section{SeÇÃo - ComentÁrios À JURISPRUdÊnCIA \\ SECTION 5 - CommentS of JuRISPRUdENCE}

ADI 6.427 DF: inovação normativa pelo Judiciário na interpretação conforme a Constituição

ADI 6,427 DF: normative innovation by the Judiciary in the interpretation according to the Constitution

Adilson Neri Pereira 
Acórdão 169/2021-TCU: um novo entendimento jurisprudencial para o cálculo da garantia adicional em contratos administrativos Judgment 169/2021-TCU: a new jurisprudential understanding for the calculation of the additional guarantee in administrative contracts

Augusto César Tavares de Lira da Cunha e Pedro Flávio Cardoso lucena .... 301

\section{SeÇÃo - EntreVISTA}

\section{Section 6 - InTERVIEW}

Entrevista com Dalmo de Abreu Dallari Interview with Dalmo de Abreu Dallari

Augusto Neves Dal Pozzo e Ricardo Marcondes Martins.

\section{Seção - Memória do Direito Administrativo}

\section{Section 7 - Retrospective of Administrative Law}

0 Conselho de Estado e o contencioso administrativo no Brasil The Council of State and administrative litigation in Brazil

Modalidades de descentralização administrativa e seu controle Modalities for administrative decentralization and its control

Celso Antônio Bandeira de Mello.

Impossibilidade de o INPS multar municípios Impossibility for the Brazilian Institute of Social Welfare to fine municipalities

Celso Antônio Bandeira de Mello.

A personalidade do Estado

The personality of the State

Oswaldo Aranha Bandeira de Mello.

Normas de Publicação para Autores de Colaboração Autoral Inédita ..... 393

Publication Guidelines for Authors to Submit Unpublished Articles ...... 395 




\section{NESTA EDIÇÃO:}

\section{ÉTICA DA DISCUSSÃO E PRINCÍPIOS DA ADMINISTRAÇÃO PÚBLICA: O ADMINISTRADOR PÚBLICO MENTIROSO E A IMPROBIDADE ADMINISTRATIVA}

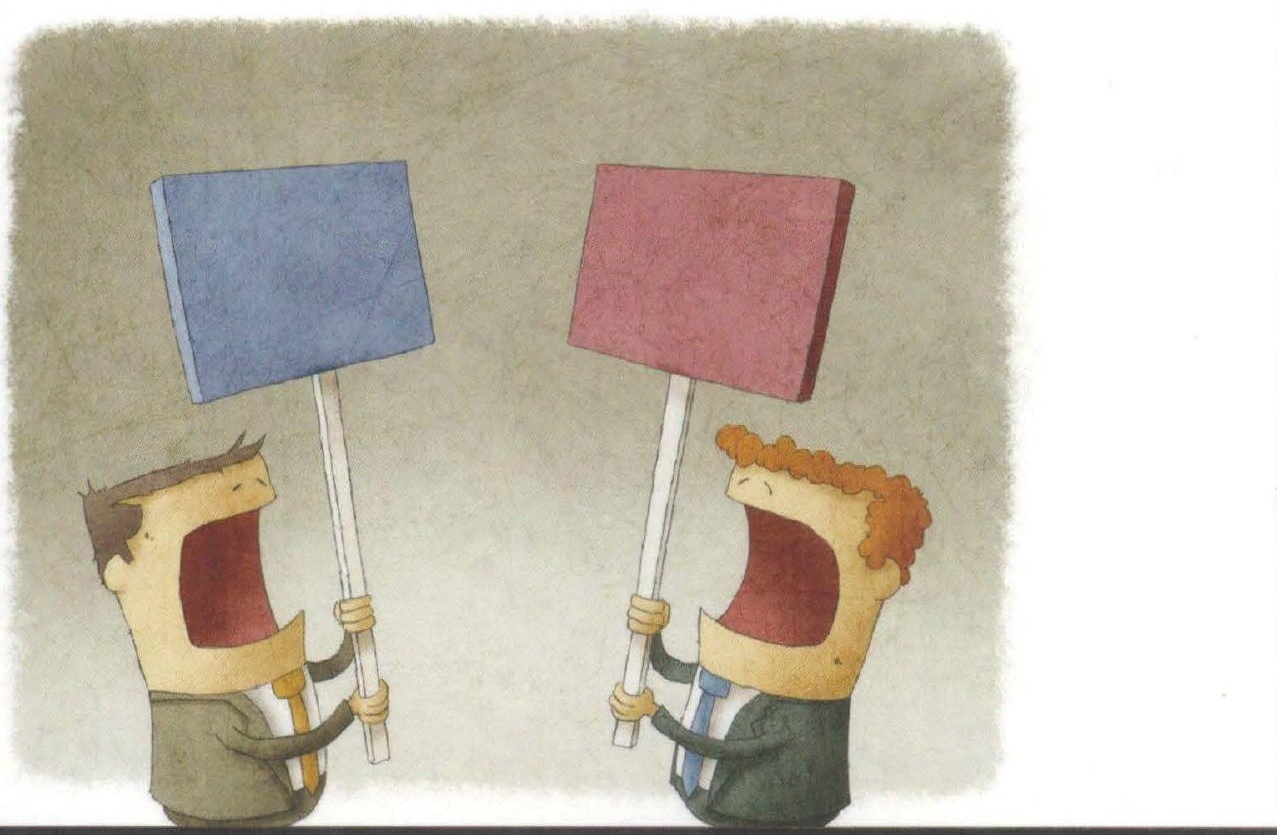

REVISTA DE DIREITO ADMINISTRATIVO, INFRAESTRUTURA, REGULAÇÃO E COMPLIANCE
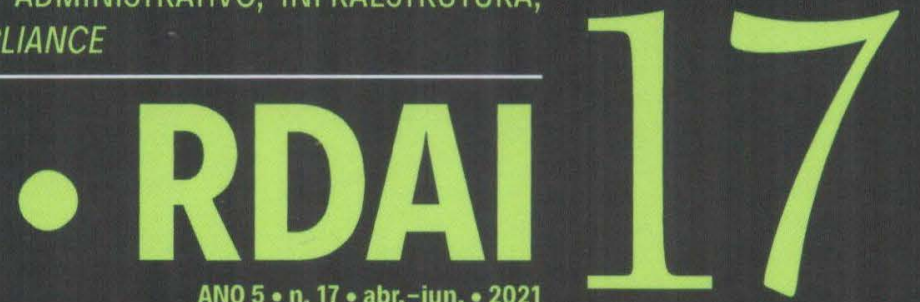

Journal of Administrative Law, Infrastructure, Regulation and Compliance

N. 5 • ISSUE $17 \cdot$ April - June $\cdot 2021$

COORDENACẢO

AUGUSTO NEVES DAL POZZO E RICARDO MARCONDES MARTINS 